\title{
BMJ Open Barriers to early diagnosis of symptomatic breast cancer: a qualitative study of Black African, Black Caribbean and White British women living in the UK
}

\author{
Claire E L Jones, ${ }^{1}$ Jill Maben, ${ }^{2}$ Grace Lucas, ${ }^{3}$ Elizabeth A Davies, ${ }^{4}$ \\ Ruth $\mathrm{H}$ Jack, ${ }^{5}$ Emma Ream $^{6}$
}

To cite: Jones CEL, Maben J, Lucas $\mathrm{G}$, et al. Barriers to early diagnosis of symptomatic breast cancer: a qualitative study of Black African, Black Caribbean and White British women living in the UK. BMJ Open 2015;5:e006944. doi:10.1136/bmjopen-2014006944

- Prepublication history for this paper is available online. To view these files please visit the journal online (http://dx.doi.org/10.1136/ bmjopen-2014-006944).

Received 17 October 2014 Revised 7 February 2015 Accepted 10 February 2015

CrossMark

For numbered affiliations see end of article.

Correspondence to

Emma Ream;

e.ream@surrey.ac.uk

\section{ABSTRACT}

Objectives: Understanding barriers to early diagnosis of symptomatic breast cancer among Black African, Black Caribbean and White British women in the UK.

Design: In-depth qualitative interviews using grounded theory methods to identify themes. Findings validated through focus groups.

Participants: 94 women aged 33-91 years; 20 Black African, 20 Black Caribbean and 20 White British women diagnosed with symptomatic breast cancer were interviewed. Fourteen Black African and 20 Black Caribbean women with $(n=19)$ and without $(n=15)$ breast cancer participated in six focus groups.

Setting: Eight cancer centres/hospital trusts in London $(n=5)$, Somerset $(n=1)$, West Midlands $(n=1)$ and Greater Manchester ( $n=1)$ during 2012-2013.

Results: There are important differences and similarities in barriers to early diagnosis of breast cancer between Black African, Black Caribbean and White British women in the UK. Differences were influenced by country of birth, time spent in UK and age. First generation Black African women experienced most barriers and longest delays. Second generation Black Caribbean and White British women were similar and experienced fewest barriers. Absence of pain was a barrier for Black African and Black Caribbean women. Older White British women ( $\geq 70$ years) and first generation Black African and Black Caribbean women shared conservative attitudes and taboos about breast awareness. All women viewed themselves at low risk of the disease, and voiced uncertainty over breast awareness and appraising non-lump symptoms. Focus group findings validated and expanded themes identified in interviews.

Conclusions: Findings challenged reporting of Black women homogenously in breast cancer research. This can mask distinctions within and between ethnic groups. Current media and health promotion messages need reframing to promote early presentation with breast symptoms. Working with communities and developing culturally appropriate materials may lessen taboos and stigma, raise awareness, increase discussion of breast cancer and promote prompt helpseeking for breast symptoms among women with low cancer awareness.

\section{Strengths and limitations of this study}

- The study was in-depth and used a large sample $(n=94)$ for qualitative research across different geographical areas.

- The effects of socioeconomic factors and ethnicity were taken into consideration by matching White British women with Black African and Black Caribbean women.

- Interview findings were further strengthened by their validation using relevant vignettes in focus groups.

- We have not fully captured the diversity of Black African women. Variation is likely to exist between countries and regions in relation to cancer-related health education, awareness, beliefs, attitudes and behaviours.

\section{BACKGROUND}

Breast cancer is the most common cancer in women and the second largest cause of death from cancer in the UK. ${ }^{1}$ UK data suggest that despite having lower breast cancer incidence rates than White British women, Black African and Black Caribbean women are more likely to be diagnosed with metastatic disease and have poorer survival outcomes than White British women. ${ }^{2}$ This may reflect the higher proportion of Black women developing triple negative breast cancer-an aggressive form of the disease associated with poorer outcomes. ${ }^{3}$ A systematic review (18 studies: 11 quantitative, 6 qualitative and 1 mixed method) identified further factors contributing to this disparity between Black and White women: lower symptom and risk factor awareness; stigma, fear and taboo; not making time for breast awareness; fear of conventional treatment; mistrust of healthcare professionals; financial burden of healthcare and inaccessibility of services. ${ }^{4}$ There was limited evidence for the influence of religiosity on delayed presentation. However, the 
review could not delineate UK perceptions and experiences, including regional differences across the UK, due to lack of UK data. Differences within and between Black ethnic groups, comparisons with White British women, differences according to place of birth and the influence of age or other factors, could not be defined due to paucity of information about samples and reporting of findings from Black women homogenously. The review could not answer how best to intervene to enhance early presentation and diagnosis of symptomatic breast cancer in the UK.

The disparity in cancer outcomes between Black and White women and paucity of in-depth qualitative research exploring barriers to help-seeking for breast cancer among Black women provided the impetus for this study. Breast awareness entails women knowing how their breasts look and feel, recognising what is normal for them and having confidence to discern unusual changes should they arise. ${ }^{5}$ Therefore, breast awareness has a key role in early presentation and it is this definition of breast awareness that was adopted for the study.

\section{STUDY AIMS}

This was a two phased study that sought to understand barriers to early diagnosis of symptomatic breast cancer among Black African, Black Caribbean and White British women. The first phase comprised interviews that aimed to explore, retrospectively, barriers to early presentation and diagnosis with breast cancer. It sought to understand similarities and differences both within and between ethnic groups. The second phase comprised focus groups conducted to validate and elaborate on findings from the interviews to provide a comprehensive account of barriers to early diagnosis with breast cancer in Black African and Black Caribbean women living in the UK.

In the UK, a national breast screening programme invites women aged 50-70 years for screening every 3 years. Women also present to healthcare professionals - typically their general practitioner (GP) - if they discover a breast change. It is this symptomatic presentation that was the focus of this study.

\section{METHODS}

The two phased study was undertaken in secondary care settings. The interview phase took place between February 2012 and March 2013. The focus group phase was conducted between August and October 2013. It was envisaged that the planned number of interviews (20 in each ethnic group) would be sufficient to attain data saturation. In-depth qualitative interviews provided detailed understanding of barriers to early diagnosis with symptomatic breast cancer in Black African and Black Caribbean women in the UK, both as unique groups, and in comparison with White British women. Subsequent focus groups with Black African and Black Caribbean women were used to validate interview findings using vignettes to provide context and stimulate discussion.

\section{Sample recruitment \\ Interview phase}

Clinical teams in five London cancer centres/hospital trusts systematically identified eligible women from clinical records and recruited them to interviews conducted face to face (figure 1). Eligible women were (1) Black African, Black Caribbean or White British (2) over

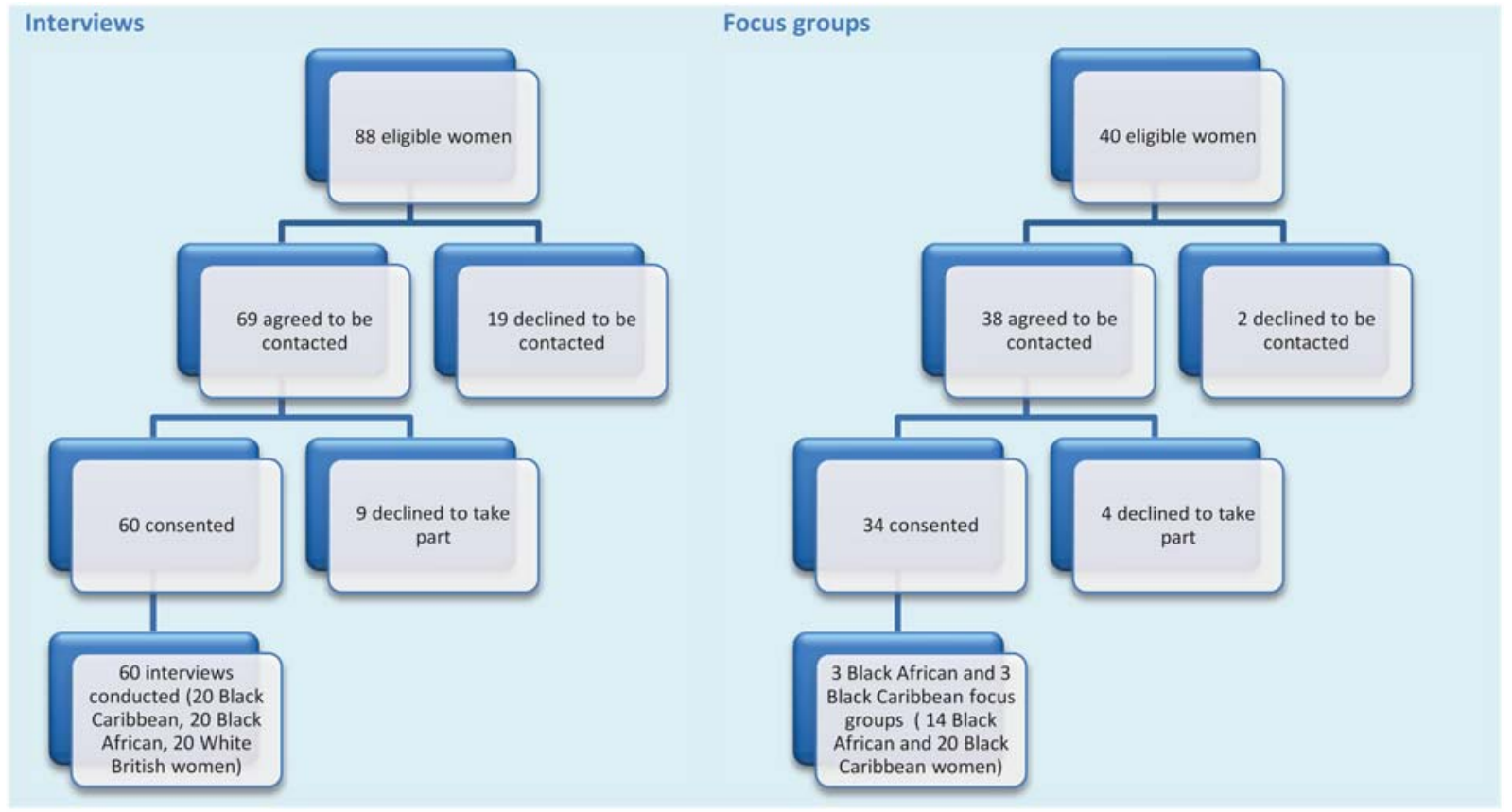

Figure 1 Recruitment flow chart. 
18 years (3) diagnosed after presenting with symptomatic primary breast cancer 2-6 months prior to recruitment. Women who did not speak English were eligible to participate but none were identified by clinical teams. Women were ineligible to participate if they presented via the National Breast Cancer Screening Programme and/or had been diagnosed with a local recurrence of breast cancer or a second primary. The study researcher (CELJ) used a questionnaire to screen women for eligibility and purposively recruit a diverse sample of differing ages, countries of birth, education and times to presentation (78\% participation rate). Delayed presentation was defined as a wait of $\geq 12$ weeks in consulting a healthcare professional about breast cancer symptoms. ${ }^{6}$ Women were identified as either first or second generation by whether they said they were born outside or inside the UK. Women self-defined their ethnicity. All women gave written informed consent. White British women were matched to the Black African and Black Caribbean samples according to age and education, when possible, to enhance comparability. However, White British women tended to be more highly educated than other groups.

\section{Focus group phase}

Clinical teams in hospital trusts in Somerset, the West Midlands and Greater Manchester systematically identified eligible women from clinical records and recruited a convenience sample of women to six focus groups. Women were contacted and recruited to focus groups either face-to-face or by telephone (figure 1). Eligible women were: (1) Black African or Black Caribbean; (2) over 18 years; (3) diagnosed with breast cancer $\geq 2$ months before the conduct of the focus groups; or (4) a family member/friend of someone diagnosed with breast cancer $\geq 2$ months prior to their conduct; and (5) English speaking.

Most patient participants identified one Black African or Black Caribbean woman each among their family and friends for the focus groups, that is, snowball sampling. CELJ used a questionnaire to screen women for eligibility (95\% participation rate) by asking about their ethnicity and age. First generation and second generation women were identified using the same process defined in the interview phase. Women self-defined their ethnicity. All women gave written informed consent. This led to six samples of four to seven women with and without breast cancer being recruited to allow comparison between intentions and behaviours. One Black African and one Black Caribbean focus group were run in each location.

\section{Data generation}

Interview phase

CELJ conducted interviews at locations chosen by the women; generally their homes. An interview guide was used to allow women to talk about salient themes in their own words and at their own pace. Themes explored included: knowledge of cancer and specifically breast cancer before diagnosis; views about breast awareness; feelings on noticing breast symptoms; determining what symptoms meant; and decisions made about, and experiences of, help-seeking. Interviews lasted $45-150 \mathrm{~min}$, were audio recorded and transcribed verbatim. Analysis ran simultaneously with interviewing. Emerging themes and their interrelationships were explored in later interviews through more focused questioning to clarify understanding.

\section{Focus group phase}

Focus groups were undertaken in hospitals by CELJ with groups of all Black African or Black Caribbean women. Patients and family/friends were in the same focus group rather than separated. Discussion was directed by a focus group guide and vignette-a digitally recorded audio story constructed from salient interview findings depicting a Black African or Black Caribbean woman's delayed presentation and diagnosis. Two vignettes were developed-one for playing in focus groups with Black African women and the other for use in focus groups with Black Caribbean women. Each provided an amalgamation of women's specific (according to particular ethnic group) experiences narrated either by a Black African or Black Caribbean actress. They covered all of the barriers to noticing breast changes, working out what such changes meant, deciding what to do and finding a way through the healthcare system identified from the interviews (box 1). Women's verbatim phrases were used to enhance authenticity. Vignettes were 4 min long and during focus group were played initially in their entirety and then by section-depicting barriers to early presentation and diagnosis-to allow detailed reflection and discussion. These sections were also printed out and given to women in the groups to help

\section{Box 1 Summary of barriers}

\section{Noticing changes}

Perceiving breast cancer information irrelevant

Low awareness of risk factors and personal risk

Low symptom awareness

Breast awareness not part of cultural norm

Concern over how to be breast aware

2. Working out what changes mean

Difficulty appraising symptoms

Not disclosing symptoms to others or disclosing to those with poor cancer knowledge

3. Deciding what to do

Fearing a cancer diagnosis

Worrying about wasting the doctor's time

Self-managing symptoms-rather than seeking help

Focusing on other things

Not knowing importance of early diagnosis

4. Finding a way through the healthcare system

Not knowing where to go

Difficulties booking GP appointments

Feeling disempowered

Difficulty organising and attending hospital appointments 
maintain focus. For example, one section relating to working out what changes meant comprised:

When I first discovered something it was a lump but it wasn't paining me so I wasn't worried. I also have children so it would have had to be something stopping me doing the things I usually do, to take time off and see the GP. I then told my husband that I noticed a lump. He said 'it's in your imagination. Put your mind off it' so I listened to him.

Women commented after each section on attitudes, beliefs and behaviours depicted. Focus groups lasted 90-120 min, were audio recorded and transcribed verbatim. A cofacilitator kept field notes and managed the audio recording equipment.

\section{Data analysis}

Interview phase

Transcribed interviews were managed using QSR NVivo (V.9). Iterative analysis, following tenets of grounded theory, ${ }^{7}$ identified themes and their inter-relationships. CELJ and GL coded transcripts line by line to categorise and describe data, then developed themes through selective and conceptual coding. Constant comparisons and contrasts enabled evolving themes to be identified and highlighted divergent perceptions and experiences, which were explored in subsequent interviews. The researcher (CELJ), principal investigator (ER) and research team (JM, GL, $\mathrm{EAD}$ and $\mathrm{RHJ}$ ) reviewed emergent findings and supported analysis. Additionally, the steering committee discussed emergent themes drawing on their experience, areas of expertise and understanding of the literature.

\section{Focus group phase}

Focus group transcripts were managed using Microsoft Excel (2007). Framework analysis integrated interview and focus group data and enabled their comparison. ${ }^{8}$ CELJ summarised focus group data into a matrix comprising core interview themes. New and conflicting findings were coded in red and incorporated into the framework.

\section{Steering committee and lay advisory group involvement}

A multidisciplinary steering committee-comprising Black African, Black Caribbean and White British women with personal experience of breast cancer who were patient advocates involved in cancer research; a healthcare professional who was a director of a Black and Minority Ethnic (BME) cancer charity; a head of research at a breast cancer charity; clinicians and academics (a consultant surgeon and senior lecturer and a consultant nurse) - advised on the study design, recruitment, emerging themes and dissemination of findings. A lay advisory group of Black African and Black Caribbean women with and without breast cancer $(n=8)$ from a BME cancer charity piloted and advised on the focus group vignettes.

\section{RESULTS}

\section{Overview of findings}

Ninety-four women participated; 20 Black African, 20 Black Caribbean and 20 White British women were interviewed and 20 Black Caribbean and 14 Black African women attended focus groups. Patterns in the data suggested variation in experience by women's ethnic group and generation (first vs second generation migrants to the UK); the results are presented to reflect this (tables 1 and 2).

There were four fundamental stages leading to diagnosis with breast cancer (figure 2). A number of barriers to early presentation and diagnosis operated within each (box 1). Findings are structured according to stages.

Self-reported times to presentation, and between presentation and diagnosis, were longest among first generation and, notably, Black African, migrants (table 1). More first generation Black African than other women were diagnosed with tumours larger than $5 \mathrm{~cm}$ and more had metastatic disease. Over half (9 out of 16) of first generation Black African women recruited to the study had metastatic disease. This suggests-although some had aggressive triple negative disease-that this group of women may have had their breast change for some time before presenting to healthcare professionals and/or had difficulty navigating the diagnostic process. However, first generation Black African women who worked in healthcare $(n=5)$ were the exception. Their exposure to the healthcare system-irrespective of occupation-appeared linked to early diagnosis. Data saturation was reached as envisaged through conduct and analysis of the 60 interviews. Further, themes identified in the interviews were confirmed and illustrated by the focus group findings. Therefore, focus group data will only be referred to if they countered or were additional to the interview findings. There was no discernible difference in the focus group data between help-seeking intentions alluded to by women without breast cancer and actual help-seeking behaviour of women with the disease.

\section{Noticing changes}

Five barriers to women noticing breast changes were revealed and influenced women differently (table 3).

\section{Perceiving breast cancer information as irrelevant}

White British women and second generation Black Caribbean women appeared receptive to information about breast cancer because the disease had personal relevance for them; many had known women with the disease. However, most first generation Black African women, some second generation Black African women and first generation Black Caribbean women, appeared less receptive towards media/health campaigns about cancer generally and specifically breast awareness, before they were diagnosed. They had limited or no personal experience of cancer. Cancer was described as taboo and stigmatised. Further, first generation Black 
Table 1 Characteristics of the 60 women with breast cancer in the interviews

\begin{tabular}{|c|c|c|c|c|c|c|}
\hline & \multirow[b]{2}{*}{$\begin{array}{l}\text { All women } \\
(\mathrm{N}=60)\end{array}$} & \multicolumn{2}{|c|}{$\begin{array}{l}\text { Black African } \\
\mathrm{N}=20\end{array}$} & \multicolumn{2}{|c|}{$\begin{array}{l}\text { Black Caribbean } \\
\mathrm{N}=20\end{array}$} & \multirow[b]{2}{*}{$\begin{array}{l}\text { White } \\
\text { British } \\
\mathrm{N}=20 \\
(\mathrm{n}=20)\end{array}$} \\
\hline & & $\begin{array}{l}\text { First } \\
\text { generation } \\
(n=16)\end{array}$ & $\begin{array}{l}\text { Second } \\
\text { generation } \\
(n=4)\end{array}$ & $\begin{array}{l}\text { First } \\
\text { generation } \\
(n=9)\end{array}$ & $\begin{array}{l}\text { Second } \\
\text { generation } \\
(n=11)\end{array}$ & \\
\hline Age years; mean (range) & $52(30-91)$ & $47(30-79)$ & $45(43-46)$ & 65 (47-91) & $45(41-57)$ & $55(31-83)$ \\
\hline \multicolumn{7}{|l|}{ Religion } \\
\hline Christian & 46 & 15 & 4 & 9 & 9 & 9 \\
\hline No religion & 11 & 0 & 0 & 0 & 0 & 11 \\
\hline $\begin{array}{l}\text { Other (Muslim and } \\
\text { Buddhist) }\end{array}$ & 3 & 1 & 0 & 0 & 2 & 0 \\
\hline \multicolumn{7}{|l|}{ Marital status } \\
\hline Single & 11 & 4 & 0 & 0 & 5 & 2 \\
\hline Cohabiting & 9 & 1 & 1 & 1 & 1 & 5 \\
\hline Married & 23 & 6 & 3 & 3 & 4 & 7 \\
\hline $\begin{array}{l}\text { Divorced/separated/ } \\
\text { widowed }\end{array}$ & 17 & 5 & 0 & 5 & 1 & 6 \\
\hline \multicolumn{7}{|c|}{ Employment (at time of interview) } \\
\hline Employed full-time & 25 & 3 & 3 & 3 & 8 & 8 \\
\hline Employed part-time & 10 & 3 & 1 & 2 & 0 & 4 \\
\hline Unemployed & 7 & 5 & 0 & 0 & 1 & 1 \\
\hline Full-time homemaker & 3 & 2 & 0 & 0 & 0 & 1 \\
\hline $\begin{array}{l}\text { Other (self-employed/ } \\
\text { student) }\end{array}$ & 5 & 1 & 0 & 0 & 2 & 2 \\
\hline Retired & 10 & 2 & 0 & 4 & 0 & 4 \\
\hline \multicolumn{7}{|l|}{ Education } \\
\hline No formal education or & 17 & 4 & 0 & 4 & 3 & 6 \\
\hline \multicolumn{7}{|l|}{ GCSE/O Level/CSE } \\
\hline A-Levels or equivalent & 21 & 10 & 0 & 3 & 5 & 3 \\
\hline Degree or equivalent & 16 & 1 & 4 & 1 & 3 & 7 \\
\hline Other & 6 & 1 & 0 & 1 & 0 & 4 \\
\hline \multicolumn{7}{|l|}{ Time to presentation } \\
\hline \multicolumn{7}{|c|}{ Self-reported time between noticing a symptom and presenting to a HCP } \\
\hline$<3$ months & 46 & 11 & 4 & 5 & 9 & 17 \\
\hline$>3$ months & 14 & 5 & 0 & 4 & 2 & 3 \\
\hline \multicolumn{7}{|c|}{ Self-reported system time between presenting to HCP and being given results } \\
\hline$<3$ months & 53 & 12 & 4 & 7 & 10 & 20 \\
\hline$>3$ months & 7 & 4 & 0 & 2 & 1 & 0 \\
\hline \multicolumn{7}{|l|}{ Breast cancer type } \\
\hline Ductal carcinoma in situ & 25 & 6 & 2 & 4 & 5 & 8 \\
\hline $\begin{array}{l}\text { Invasive (or infiltrating) } \\
\text { ductal carcinoma }\end{array}$ & 25 & 7 & 2 & 3 & 4 & 9 \\
\hline $\begin{array}{l}\text { Invasive (or infiltrating) } \\
\text { lobular carcinoma }\end{array}$ & 2 & 0 & 0 & 0 & 0 & 2 \\
\hline $\begin{array}{l}\text { Inflammatory breast } \\
\text { cancer }\end{array}$ & 1 & 0 & 0 & 0 & 0 & 1 \\
\hline $\begin{array}{l}\text { Triple-negative breast } \\
\text { cancer }\end{array}$ & 7 & 3 & 0 & 2 & 2 & 0 \\
\hline \multicolumn{7}{|l|}{ Staging $(T N M)^{\star}$} \\
\hline $\mathrm{T} 1$ & 36 & 5 & 3 & 4 & 7 & 17 \\
\hline T2 & 14 & 3 & 1 & 3 & 4 & 3 \\
\hline T3 & 7 & 5 & 0 & 2 & 0 & 0 \\
\hline $\mathrm{T} 4$ & 3 & 3 & 0 & 0 & 0 & 0 \\
\hline No & 35 & 6 & 3 & 4 & 7 & 15 \\
\hline $\mathrm{N} 1$ & 16 & 3 & 1 & 4 & 3 & 5 \\
\hline N2 & 4 & 2 & 0 & 1 & 1 & 0 \\
\hline N3 & 5 & 5 & 0 & 0 & 0 & 0 \\
\hline
\end{tabular}


Table 1 Continued

\begin{tabular}{|c|c|c|c|c|c|c|}
\hline & \multirow[b]{2}{*}{$\begin{array}{l}\text { All women } \\
(\mathrm{N}=60)\end{array}$} & \multicolumn{2}{|c|}{$\begin{array}{l}\text { Black African } \\
\mathrm{N}=20\end{array}$} & \multicolumn{2}{|c|}{$\begin{array}{l}\text { Black Caribbean } \\
\mathrm{N}=20\end{array}$} & \multirow[b]{2}{*}{$\begin{array}{l}\text { White } \\
\text { British } \\
\mathrm{N}=20 \\
(\mathrm{n}=20)\end{array}$} \\
\hline & & $\begin{array}{l}\text { First } \\
\text { generation } \\
(n=16)\end{array}$ & $\begin{array}{l}\text { Second } \\
\text { generation } \\
(n=4)\end{array}$ & $\begin{array}{l}\text { First } \\
\text { generation } \\
(n=9)\end{array}$ & $\begin{array}{l}\text { Second } \\
\text { generation } \\
(n=11)\end{array}$ & \\
\hline MO & 48 & 7 & 4 & 7 & 10 & 20 \\
\hline M1 & 12 & 9 & 0 & 2 & 1 & 0 \\
\hline \multicolumn{7}{|l|}{ Tumour size* } \\
\hline$\leq 2 \mathrm{~cm}$ & 36 & 5 & 3 & 4 & 7 & 17 \\
\hline$>2 \mathrm{~cm}<5 \mathrm{~cm}$ & 14 & 3 & 1 & 3 & 4 & 3 \\
\hline$\geq 5 \mathrm{~cm}$ & 10 & 8 & 0 & 2 & 0 & 0 \\
\hline
\end{tabular}

*TNM breast cancer staging and tumour size ranges taken from Cancer Research UK 2012.

GCSE, General Certificate of Secondary Education; HCP, healthcare professional; $N=$ number of participants; $n=$ number of participants included in group.

African women were more familiar with other diseases, including HIV/AIDS, which were more prevalent and featured prominently in public health campaigns in the countries these women migrated from. Consequently, cancer often 'had no meaning' for Black African migrants unless they knew someone with the disease.

\section{Low awareness of risk factors and personal risk}

White British women and second generation Black Caribbean women seemed familiar with breast cancer but often believed they were not at risk of the disease as they had no family history of it. Younger women in these groups appeared less breast aware than women aged over 50-they rightly believed they had limited risk of developing the disease due to their age and therefore checked their breasts infrequently. White British participants aged over 70 years often believed older women could not develop breast cancer. Most Black African and first generation Black Caribbean women irrespective of age generally thought they were not at risk. They also believed breast cancer was a 'White woman's disease' and assumed that having no family history, feeling healthy, being young and having small breasts made it impossible for them to develop breast cancer. Some associated negative cervical screening results with having 'little chance of getting cancer' and so were not breast aware. Exceptions were women who knew people with cancer generally and who worked in healthcare.

\section{Low symptom awareness}

White British and second generation Black Caribbean women appeared to have greatest knowledge of breast cancer symptoms and typically determined quickly 'any difference' in their breasts. However, perceived 'overemphasis on lumps' in the media meant that some did not realise the significance of non-lump symptoms. Likewise, many first generation Black Caribbean and second generation Black African women were unaware of non-lump symptoms. In comparison, first generation Black African women had poor-if any-knowledge of breast cancer symptoms unless they had watched television health programmes where breast cancer had featured.

\section{Breast awareness not part of cultural norm}

White British women and second generation Black Caribbean women generally believed breast awareness was important for health. However, some White British participants aged over 70 years reported different views, reporting feeling awkward touching their bodies or believed being breast aware was 'looking for trouble'. In this respect they appeared similar to Black African women and first generation Black Caribbean women. Additionally, first generation Black African women believed feeling breasts for changes might cause breast cancer.

\section{Concern over how to be breast aware}

Women from all ethnic groups were concerned over actions to take in being breast aware. Many believed it involved a formal checking procedure and felt unconfident regarding how their breasts should feel.

\section{Working out what changes mean}

Two barriers to women interpreting symptoms as concerning were revealed and were experienced differently by ethnic group and women's time spent in the UK (table 4).

\section{Difficulty appraising symptoms}

Women across the sample who detected subtle changes (eg, 'slight hardness') reported difficulties determining their importance. Misattribution of non-lump symptoms (to menopause, menstrual cycle, age, stress and breast injury) contributed to delay in help-seeking. In White British and second generation Black Caribbean women, this appeared more likely when non-lump symptoms failed to match their mental images of them. In comparison, first generation Black African and Black Caribbean women had low awareness of non-lump symptoms. Additionally, Black Caribbean women and a 
Table 2 Characteristics of the 34 women with and without breast cancer in the focus groups

\begin{tabular}{|c|c|c|c|c|c|}
\hline & \multirow[b]{2}{*}{$\begin{array}{l}\text { All women } \\
(\mathrm{N}=34)\end{array}$} & \multicolumn{2}{|l|}{$\begin{array}{l}\text { Black African } \\
\mathrm{N}=14\end{array}$} & \multicolumn{2}{|c|}{$\begin{array}{l}\text { Black Caribbean } \\
\mathrm{N}=20\end{array}$} \\
\hline & & $\begin{array}{l}\text { First generation } \\
(n=12)\end{array}$ & $\begin{array}{l}\text { Second } \\
\text { generation }(n=2)\end{array}$ & $\begin{array}{l}\text { First } \\
\text { generation } \\
(n=5)\end{array}$ & $\begin{array}{l}\text { Second } \\
\text { generation }(n=15)\end{array}$ \\
\hline Age (years; mean (range) & $55(33-68)$ & $43(40-55)$ & $40(39-40)$ & $65(45-68)$ & $50(33-60)$ \\
\hline \multicolumn{6}{|l|}{ Religion } \\
\hline Christian & 30 & 11 & 2 & 4 & 13 \\
\hline No religion & 1 & 0 & 0 & 0 & 1 \\
\hline $\begin{array}{l}\text { Other (Muslim and } \\
\text { Buddhist) }\end{array}$ & 3 & 1 & 0 & 1 & 1 \\
\hline \multicolumn{6}{|l|}{ Marital status } \\
\hline Single & 6 & 2 & 1 & 0 & 3 \\
\hline Cohabiting & 11 & 5 & 1 & 0 & 5 \\
\hline Married & 11 & 3 & 0 & 3 & 5 \\
\hline $\begin{array}{l}\text { Divorced/separated/ } \\
\text { widowed }\end{array}$ & 6 & 2 & 0 & 2 & 2 \\
\hline \multicolumn{6}{|c|}{ Employment (at time of interview) } \\
\hline Employed full-time & 14 & 5 & 2 & 0 & 7 \\
\hline Employed part-time & 8 & 3 & 0 & 2 & 3 \\
\hline Unemployed & 2 & 1 & 0 & 0 & 1 \\
\hline Full-time homemaker & 2 & 1 & 0 & 0 & 1 \\
\hline $\begin{array}{l}\text { Other (self-employed/ } \\
\text { student) }\end{array}$ & 2 & 0 & 0 & 1 & 1 \\
\hline Retired & 6 & 2 & 0 & 2 & 2 \\
\hline \multicolumn{6}{|l|}{ Education } \\
\hline No formal education or & 3 & 2 & 0 & 1 & 0 \\
\hline \multicolumn{6}{|l|}{ GCSE/O Level/CSE } \\
\hline A-Levels or equivalent & 15 & 6 & 0 & 3 & 6 \\
\hline Degree or equivalent & 15 & 4 & 2 & 1 & 8 \\
\hline Other & 1 & 0 & 0 & 0 & 1 \\
\hline \multicolumn{6}{|c|}{ Number of women with and without breast cancer } \\
\hline With breast cancer & 19 & 6 & 2 & 5 & 6 \\
\hline Without breast cancer & 15 & 6 & 0 & 3 & 6 \\
\hline \multicolumn{6}{|c|}{ Time to presentation for women with breast cancer } \\
\hline \multicolumn{6}{|c|}{ Self-reported time between noticing a symptom and presenting to a HCP } \\
\hline$<3$ months & 14 & 3 & 2 & 3 & 6 \\
\hline$>3$ months & 5 & 3 & 0 & 2 & 0 \\
\hline \multicolumn{6}{|c|}{ Self-reported system time between presenting to $\mathrm{HCP}$ and being given results } \\
\hline$<3$ months & 16 & 3 & 2 & 5 & 6 \\
\hline$>3$ months & 3 & 3 & 0 & 0 & 0 \\
\hline
\end{tabular}

minority of White British women with lumpy breasts described difficulties differentiating between concerning and 'normal' lumps.

All women reported that they had been attentive to worsening and persistent symptoms and those they perceived as 'not normal' or having no explanation. However, first generation Black African and Black
Caribbean women seemed most likely to tolerate or ignore symptoms if they were not bothersome and did not affect daily functioning. Most Black African and Black Caribbean women assumed cancer would be painful. Those who noticed a painless lump often took longer to present compared to women who felt associated pain.

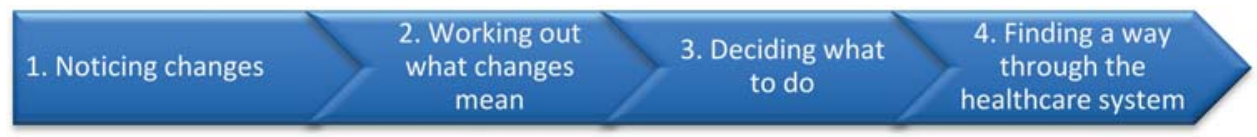

Figure 2 Journey to diagnosis with symptomatic breast cancer in Black African, Black Caribbean and White British women. 


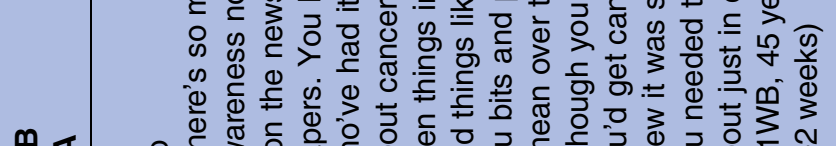

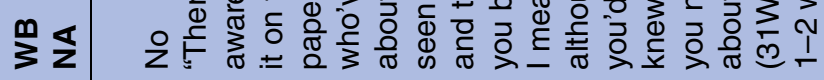

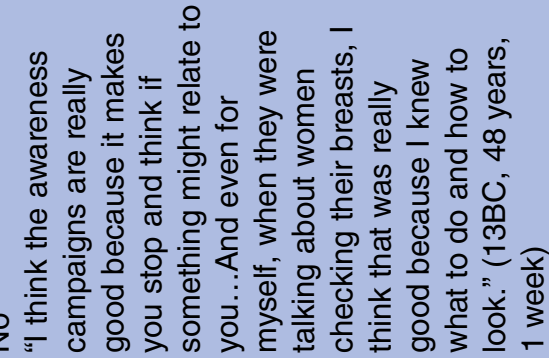

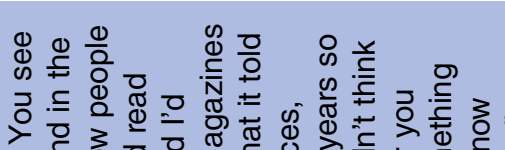

뒨 $2=0$ ठ

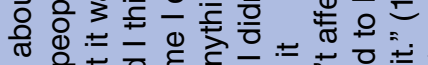

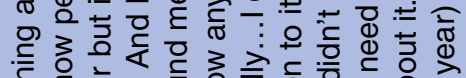

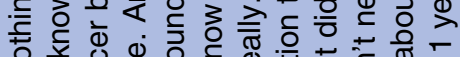

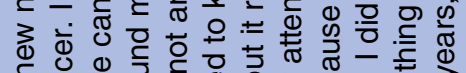

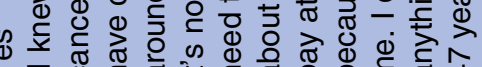

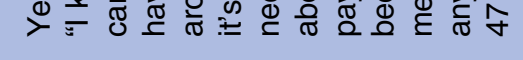

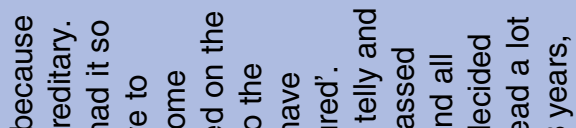

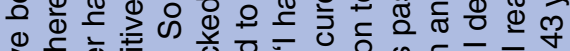

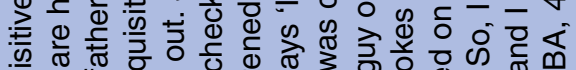

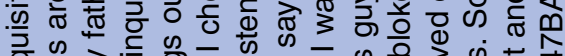

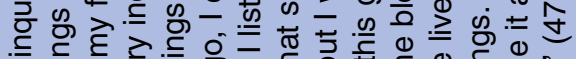

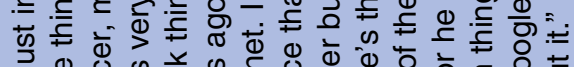

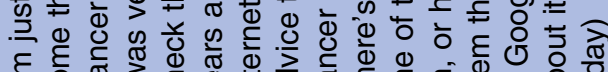

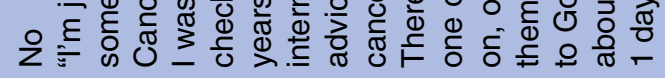

ह

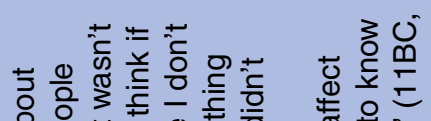

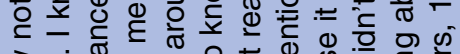

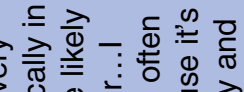

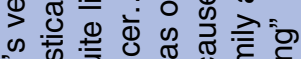

उ के

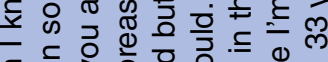

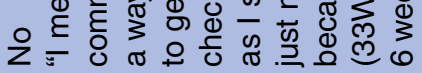

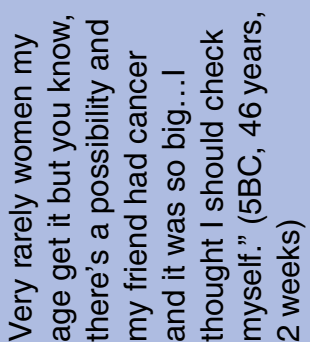

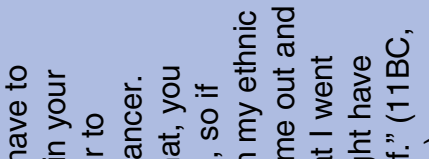

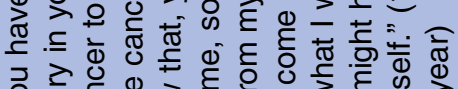

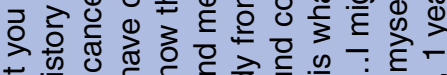

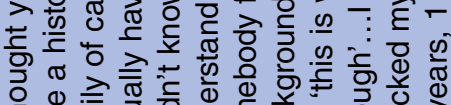

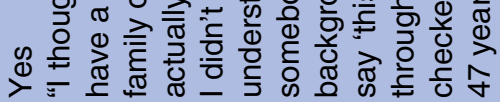

흔

『

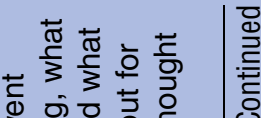

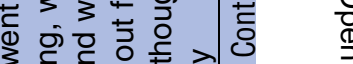

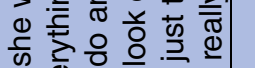

@)

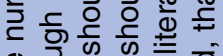

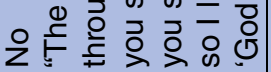

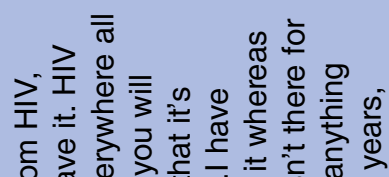

苂

을

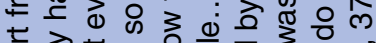

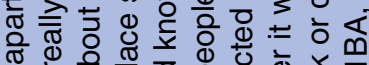

ণิ

ชీ

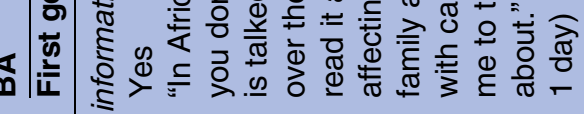

ఫิ

敢

$\frac{0}{0}$

을

政

这造

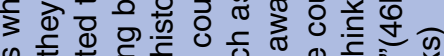

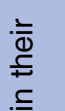

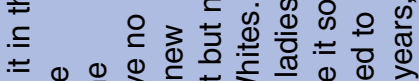

ఏ

๙

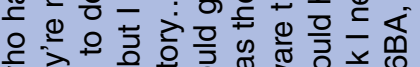

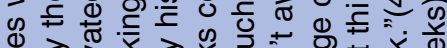

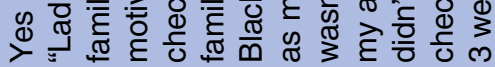

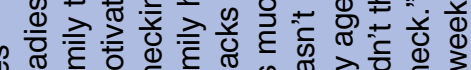

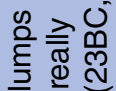

亏亏

ते बै

3 인

इ के के

入

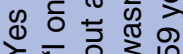

응

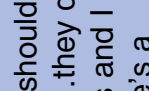

ㅇํㅇำ

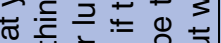

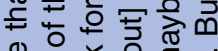

ब。

交

๒

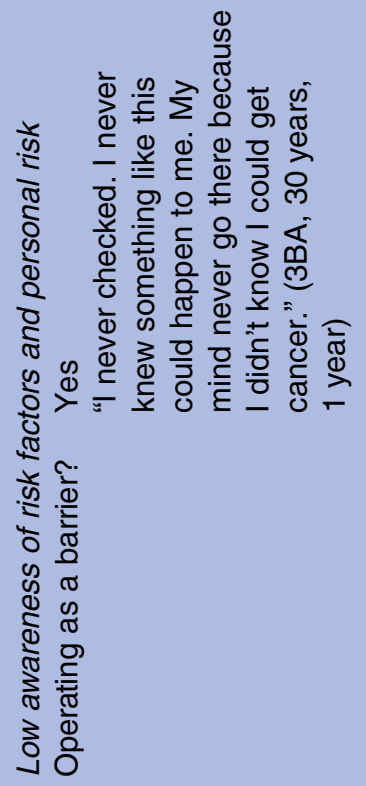

๘

这 蛋

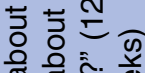

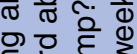

衰宁 至

흐름

उ㐫造离

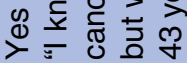

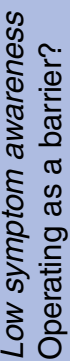

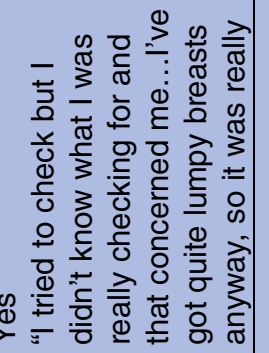

응 


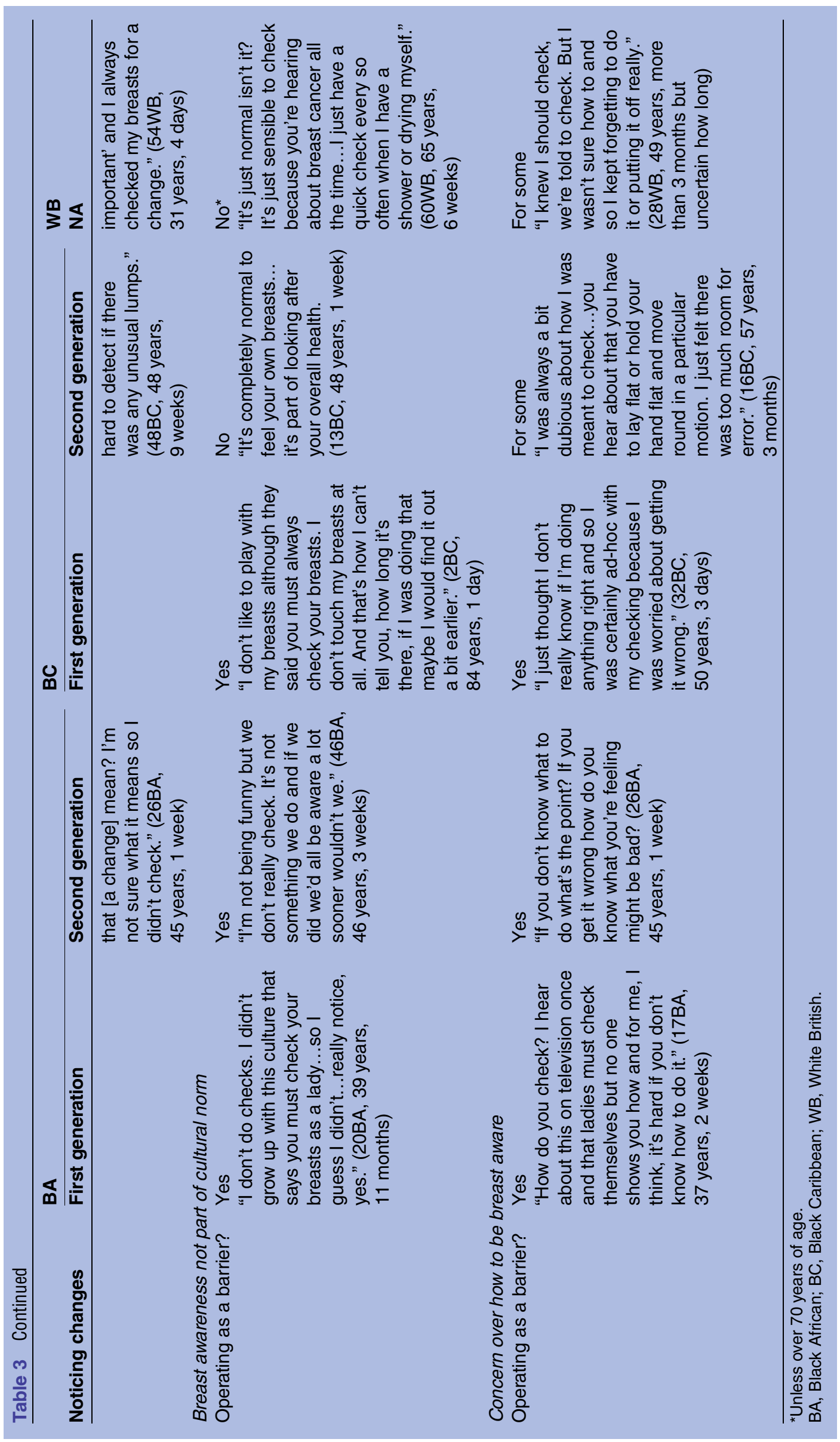




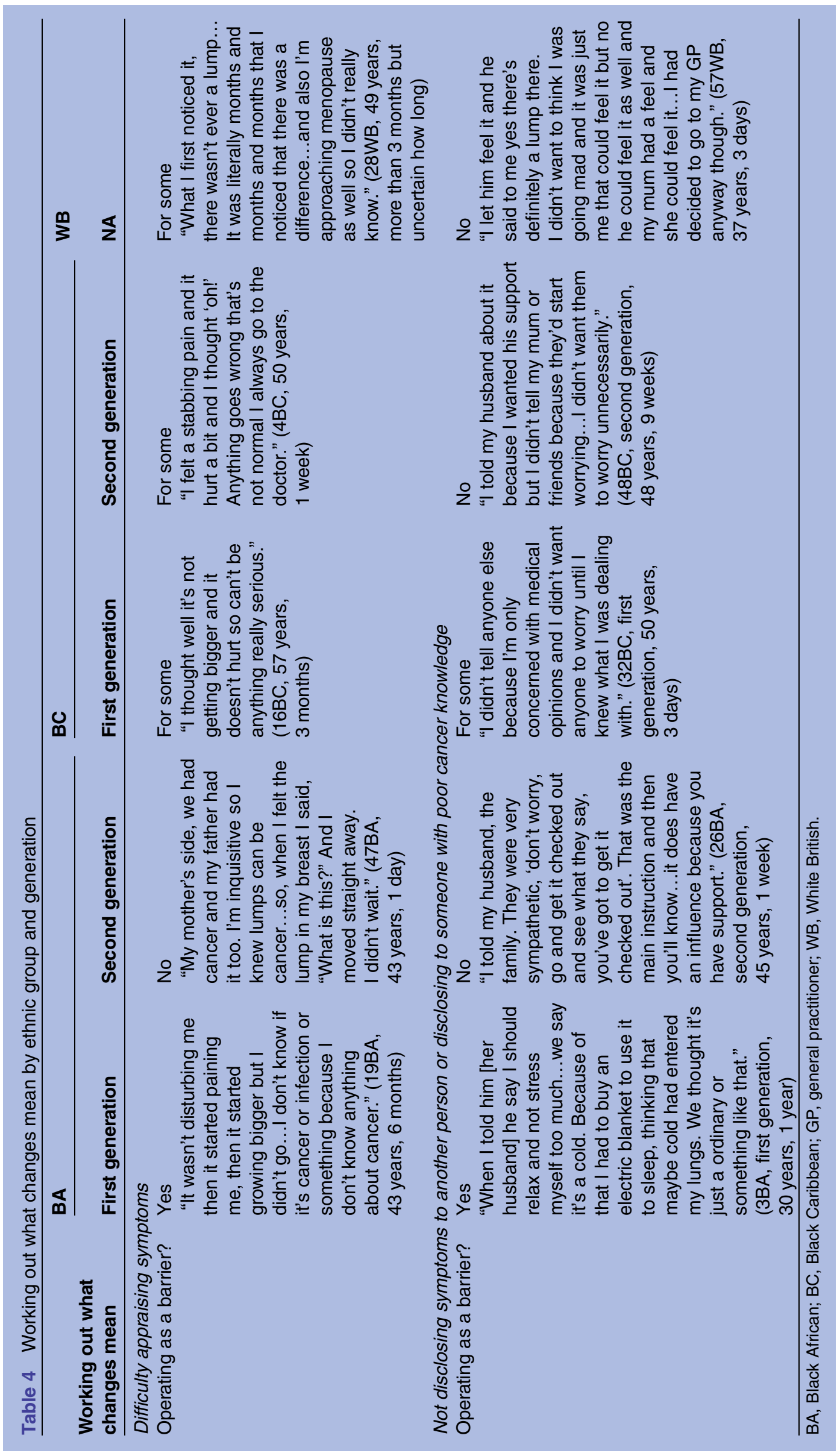


Not disclosing symptoms to others or disclosing to those with poor cancer knowledge

Disclosing symptoms appeared to promote prompt help seeking for breast changes. However, who women disclosed to was equally important. White British women and second generation Black Caribbean and Black African women were encouraged by family and friends to seek help. However, first generation Black African and Black Caribbean women reported they were not always encouraged to do so, which contributed to delay. Exceptions were women who disclosed to family or friends who either worked in healthcare or had themselves experienced a breast lump.

\section{Deciding what to do}

Table 5 summarises factors influencing women's helpseeking behaviours on noticing breast changes.

\section{Fearing a cancer diagnosis}

Fear appeared to influence help seeking for breast changes in different ways. Wanting to 'rule cancer out' rather than 'worrying unnecessarily' motivated most White British, Black Caribbean and second generation Black African women to seek help. However, fear and 'not wanting to face the possibility of breast cancer' contributed to delay among first generation Black African women whose family and friends had died from the disease. Similarly, a minority of Black Caribbean and White British women said they delayed seeking help because they believed they definitely had cancer and were anxious about how advanced it might be.

\section{Worrying about wasting doctors' time}

Irrespective of their ethnicity, women generally sought help quickly if they felt their GP or healthcare professional had been sympathetic at previous presentations with breast changes and they had been satisfied with their care. First generation and second generation Black Caribbean and first generation Black African women seemed most worried about wasting their GP's time, particularly when they perceived their GP had been dismissive or unsympathetic about previous health issues including breast changes. A minority of White British and second generation Black Caribbean women who had previously been diagnosed with benign breast disease were concerned about wasting their doctors' time and typically did not seek help with any urgency. Second generation Black African women did not report concern over wasting doctors' time.

\section{Self-managing symptoms rather than seeking help}

Most White British and second generation Black Caribbean and Black African women monitored their symptoms for a short period (range 1 day- 6 weeks) before seeking help. However, self-treating symptoms with conventional medicines (eg, painkillers) contributed to some first generation Black Caribbean women delaying presentation (range 1 day-2 years). Three first generation Black African women used prayer and alternative medicine before seeking medical help (range 2 weeks-1 year).

\section{Focusing on other things}

Competing priorities (eg, work and childcare commitments) contributed to help-seeking delay among a minority of women. Some White British women, first generation and second generation Black Caribbean women and a minority of first generation Black African woman reported delay in presenting with what they believed was breast cancer because of difficult life events (eg, depression, relationship breakdown and redundancy). However, a minority of first generation Black African and Black Caribbean women with delayed presentation appeared to genuinely believe their symptoms were unimportant within their very busy lives.

\section{Not knowing importance of early diagnosis}

Most White British and Black Caribbean women voiced the importance of early diagnosis for cancer survival and this motivated them to seek help. Conversely, most first generation Black African women were unaware of the importance of early diagnosis. Some also believed cancer remained within the breast rather than metastasising.

\section{Finding a way through the healthcare system}

Four barriers to women travelling effectively through the healthcare system were revealed in the data; again, some were experienced differently across the sample (table 6).

\section{Not knowing where to go}

All White British, Black Caribbean and second generation Black African women presented to a GP or walk-in clinic. However, many first generation Black African women presented at accident and emergency (A\&E) departments and one waited to be contacted by the National Health Service (NHS) breast screening programme because they were unsure where to present with breast changes. All but one of the Black African women presenting to A\&E were told by staff to contact their GP. The exception was a woman with metastatic disease who was admitted. However, some did not act quickly on this instruction as they did not believe it was important to do so.

\section{Difficulty booking GP appointments}

All women expressed difficulty seeing their GP, reporting: challenges in booking appointments; discomfort with disclosing symptoms to receptionists; inconvenient surgery opening hours; and limited emergency appointments. Most overcame these issues and were seen rapidly. Exceptions were women in senior/professional roles who described difficulty taking time off work, and first generation Black African women with work and childcare responsibilities (eg, agency work, childcare costs and not having a partner to look after children). Black African and Black Caribbean women in focus 
$\stackrel{m}{3} \frac{\Phi}{2}$

㐘

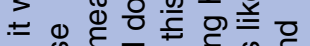

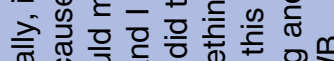

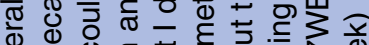

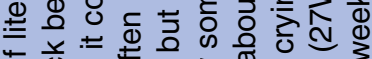

to 0 .

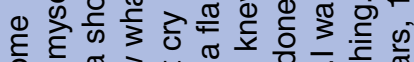

它

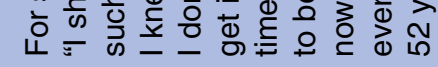

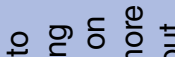

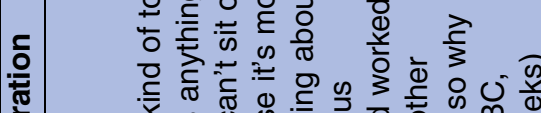

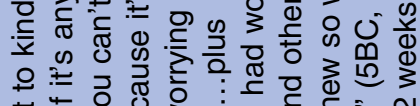
을

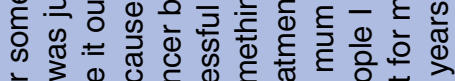

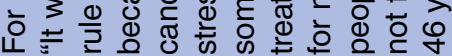

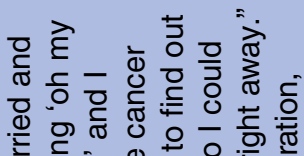

б

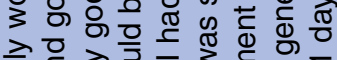

o

5

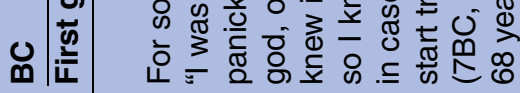

을

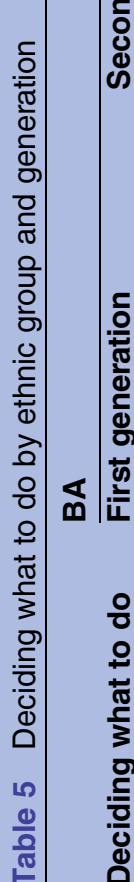

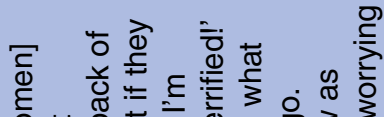

Oे क o

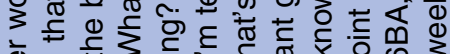

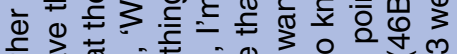

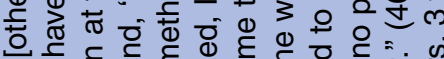

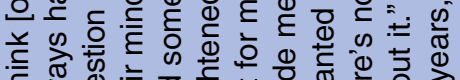

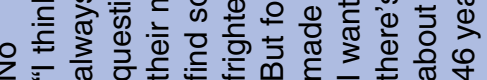

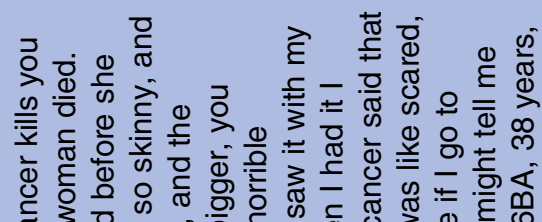

đ广.

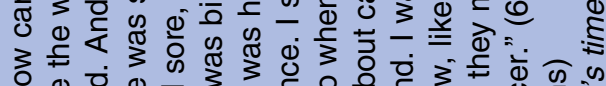

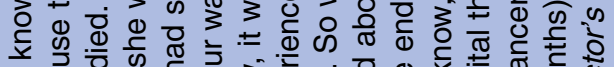

踏 क

⿻

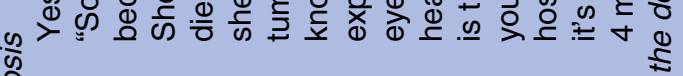

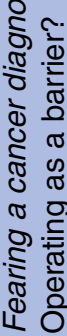

$\pm$

항ㅇㅇ

ठे

क.

ه

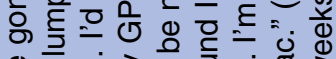

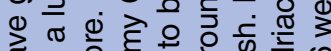

船

응

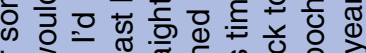

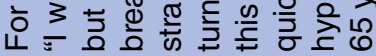

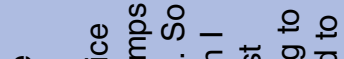

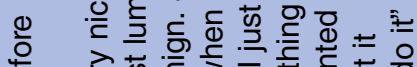

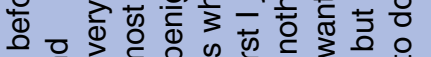

का का का

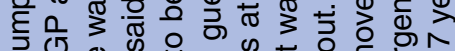

이의 0

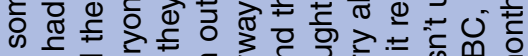

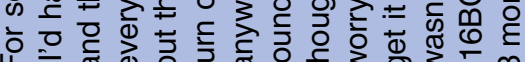

政

을

क

类

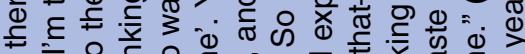

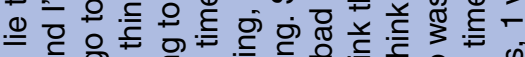

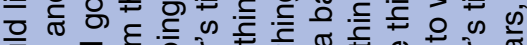

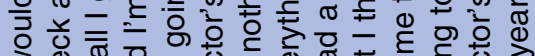

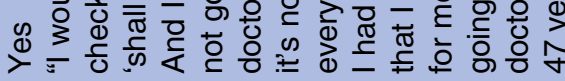

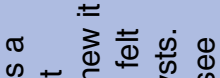

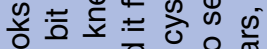

응

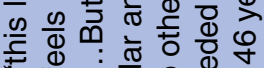

t:

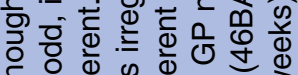

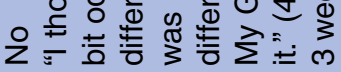

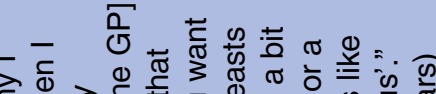

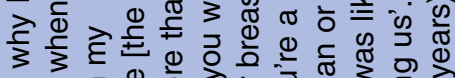

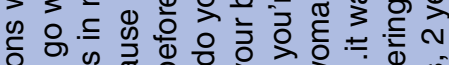

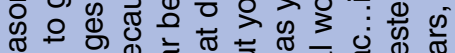

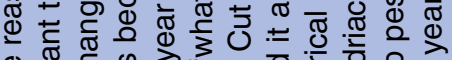

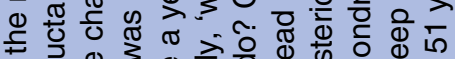

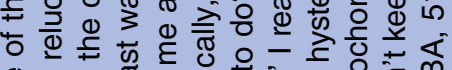

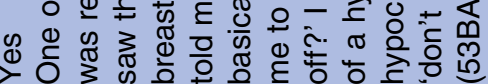

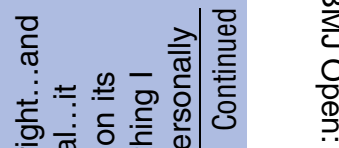

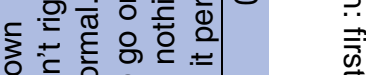

잉

ब

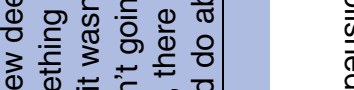

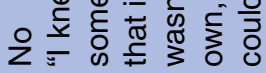

$\stackrel{\infty}{ \pm}$

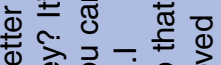

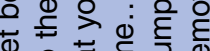

बㅇㅇㅇㅝ

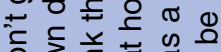

응 긍

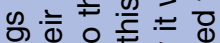

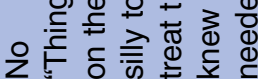

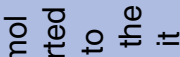

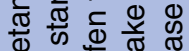

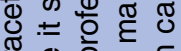

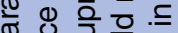

응

क्षे

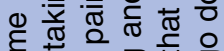

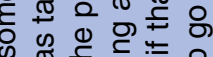

क

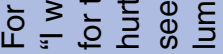

这 $₫$

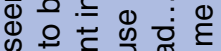

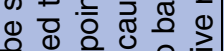

年

응

舟

西

3 3 3 要

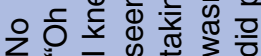

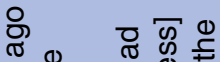

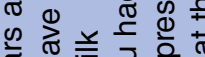

ब。

>

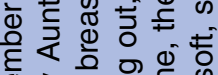

ब्र $\overrightarrow{0}$

๑ ब.

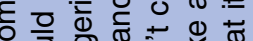
क ठำ 든

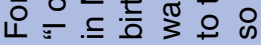

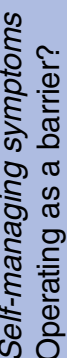




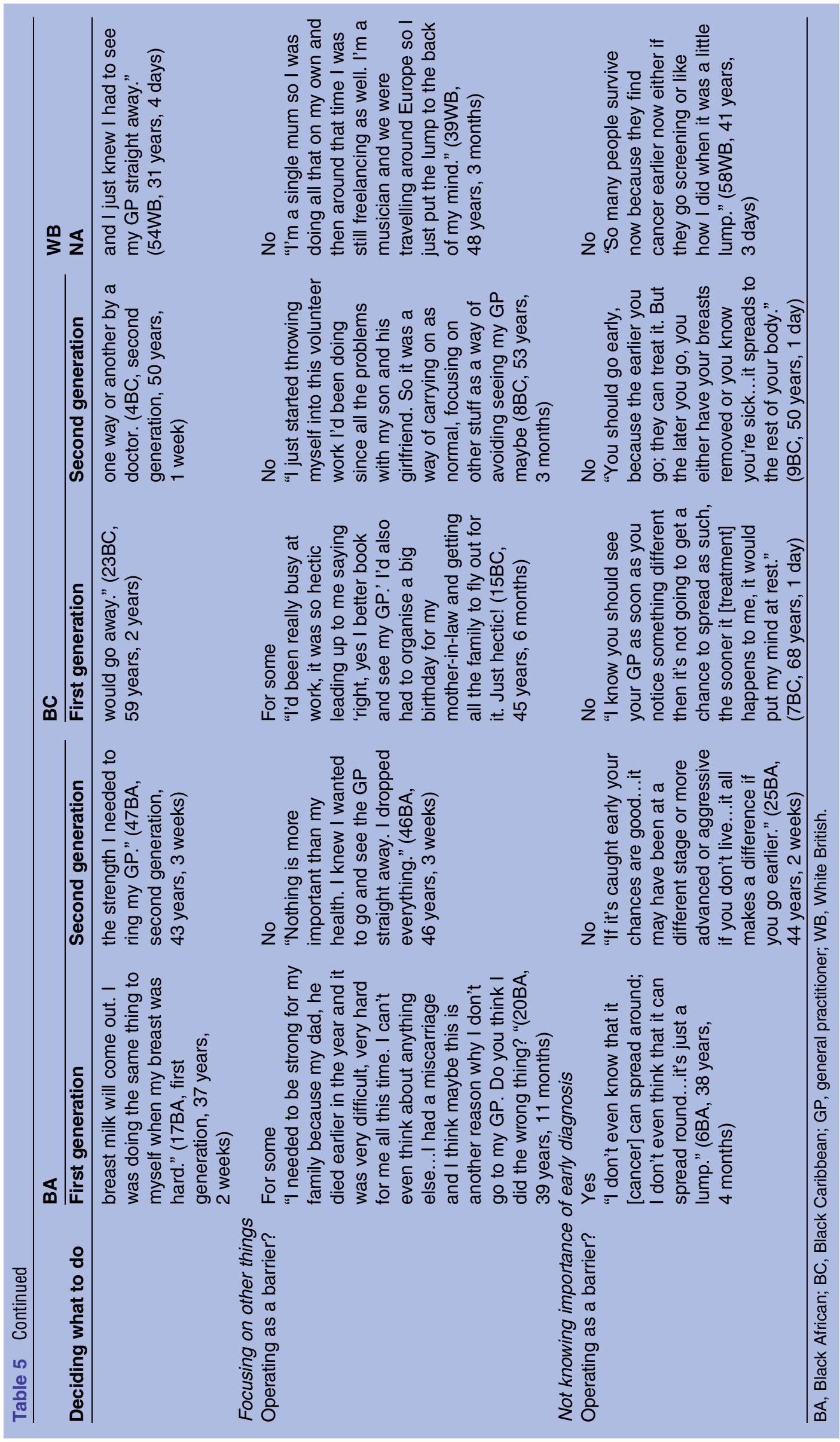




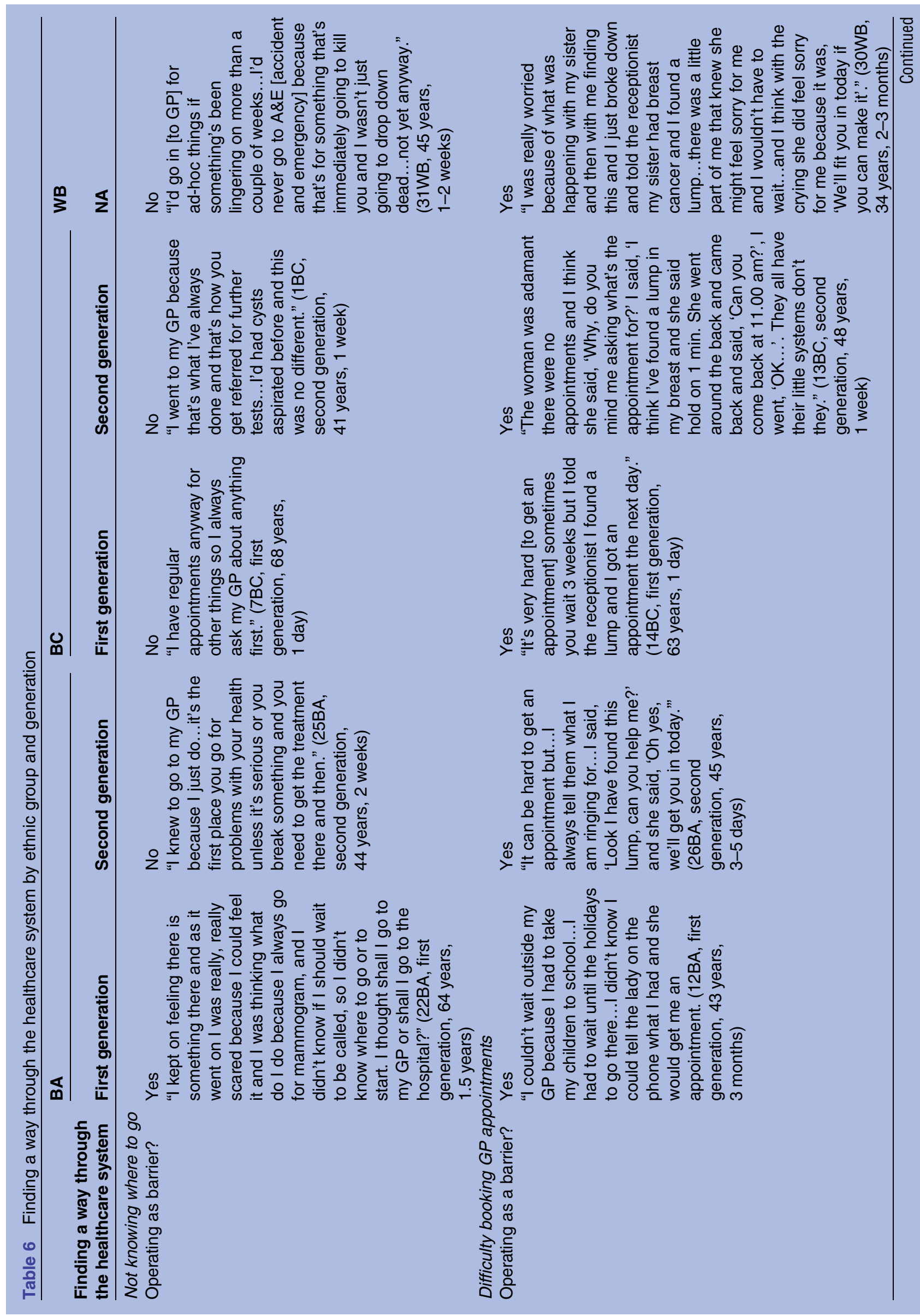




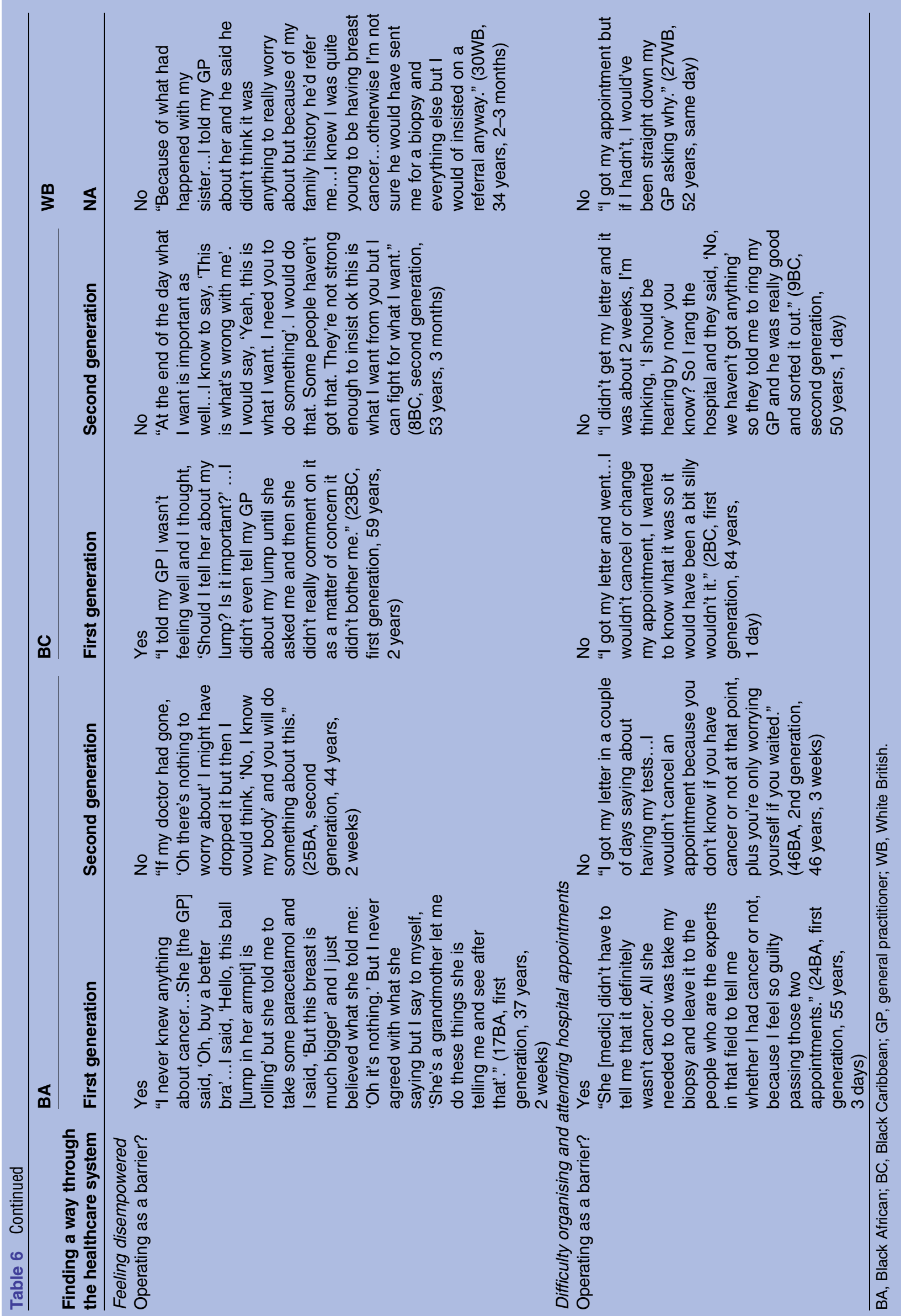


groups outside London did not experience these difficulties.

\section{Feeling disempowered}

White British and second generation Black Caribbean and Black African women appeared more empowered than other women (eg, they were aware of national guidelines for referrals of suspected breast cancer and said they felt comfortable querying GP diagnoses and seeking a second opinion). Most also said they knew GPs were not specialists. However, first generation Black African and Black Caribbean women were less forthright with professionals. Some experienced what they perceived as dismissive behaviour from their GP, including: not conducting breast examinations; telling them their breast change was normal; advising them to take painkillers despite not having pain; prescribing antibiotics; advising them to buy a better bra; or asking whether they had been touching their breasts to cause symptoms. They reported feeling uncomfortable questioning their GP, believing doctors were 'experts' and patients, 'little people'. Consequently, some reported delaying seeing their GP a second time. Some second generation Black Caribbean women in the focus groups believed Black patients, particularly those with strong accents, received a different level of care from White doctors than White patients.

\section{Difficulty organising and attending hospital appointments}

White British, first generation and second generation Black Caribbean and second generation Black African women appeared proactive in their care. They queried breast clinic appointments if these did not arrive and attended results appointments. Conversely, many first generation Black African women seemed unaware they could contact the hospital or their GP if they did receive appointments for investigations or results. Some repeatedly cancelled results appointments believing hospital staff would contact them if they had cancer.

\section{DISCUSSION}

\section{Summary of findings}

Our findings suggest clear and important distinctions between and within ethnic groups as well as similarities not previously identified. Women's nationality and country of birth appeared to be important factors influencing knowledge, attitudes and behaviours. Some regional differences also appeared to exist regarding ease of booking GP appointments and there was some suggestion regarding variability of treatment by ethnicity in one location. Second generation Black Caribbean women appeared similar to White British women regarding barriers to early presentation and diagnosis with breast cancer, and their ability to overcome them. However, first generation Black African women seemed the most disempowered group and appeared particularly vulnerable to delays. They typically had low breast awareness and symptom knowledge and were unaware of the importance of early diagnosis or how to negotiate the UK healthcare system. Older White British women ( $\geq 70$ years) and first generation Black African and Black Caribbean women shared conservative attitudes and taboos about breast awareness. All women tended to view themselves at low risk of the disease, and voiced uncertainty over how to be breast aware and had difficulty appraising non-lump symptoms.

\section{Conceptualisation of illness}

Consistent with previous studies, misattribution of symptoms appeared to influence longer times to presentation among all women. ${ }^{9}$ First generation Black African and Black Caribbean women who experienced painless breast changes, women with non-lump changes and those with lumpy breasts seemed most likely to misattribute or ignore symptoms. Early stage cancer does not figure in the popular experience in African countries. ${ }^{10} 11$ Rapidly developing infectious diseases predominate, which may explain why first generation Black African women tended to experience difficulties appraising their breast cancer symptoms. Therefore, health professionals may consider drawing out and taking into account the fact that conceptualisation of cancer symptoms may vary between ethnic groups. $^{12}$

\section{Rethinking health promotion and media messages}

Our findings suggest it may be necessary for health promotion and media messages about breast cancer to be modified. It is very important that GPs inform women that family history is a strong risk factor for breast cancer. However, women also need to be aware that the majority of women diagnosed with breast cancer do not have a family history of the disease. This is important for health promotion messages as our findings suggest that women without a family history of breast cancer may not seek help in a timely fashion, should symptoms of the disease arise. Further, there appears to be an emphasis in the media on lumps and a resultant lack of clarity over non-lump symptoms, which may influence health behaviours. Lack of understanding about what to do to be breast aware appeared to be a barrier for all ethnic groups in this study. While breast self-examination does not prevent deaths due to breast cancer, ${ }^{13}$ women are encouraged to be breast aware. ${ }^{14}$ Unfortunately, women may not always be mindful of what being breast aware means. Logically, if women are unaware of how their breasts typically look and feel, they could find it difficult to recognise subtle changes and might only detect them when the disease is relatively advanced. ${ }^{15}$ Therefore, health messages might be needed outlining the benefits of early diagnosis and specifically what breast awareness entails. ${ }^{16}$ It may be important to publicise increased breast cancer survival rates $^{17}$ because some studies suggest that fear-led messages communicating negative consequences of delayed presentation may lead to delay in women who wish to avoid a cancer diagnosis. ${ }^{18}$ 
However, other research indicates that relaying evidencebased risk for colorectal cancer through motivational interviewing might be highly effective. ${ }^{19}$ This suggests that how messages are delivered (eg, through motivational interviewing) as well as their content may influence behaviour. Our findings have identified potential key differences in breast cancer perceptions and behaviours between and within ethnic groups. Therefore, health promotion messages may need to be framed accordingly. ${ }^{20}$

\section{Understanding diverse communities' needs}

Difficulty in booking and attending appointments appeared to contribute to longer presentation times in London for professional women and for casual workers (frequently first generation Black African women). Limited access to healthcare services is a well-known barrier to help-seeking. ${ }^{21}$ Increased GP practice opening hours, ${ }^{22}$ community-based health clinics ${ }^{23}$ and telephone consultations, ${ }^{24}$ may prove effective at meeting need, particularly in London where access can be more challenging than in the rest of England. ${ }^{25}$ Further, opportunities to educate first generation Black African women about the importance of early diagnosis or to refer them directly onto breast specialists may have been missed by A\&E staff. Black Caribbean women in the Somerset focus group perceived ethnic disparities in healthcare. This may reflect the relatively small Black population in that geographical location; staff may have limited awareness of women's culturally-related needs. Further, Black African women seemed most likely to report provider delays. Disparities could be addressed through a framework of cultural interventions-which could include appropriate health educational materials tailored to different ethnic groups' needs and further training for healthcare professionals on the knowledge, attitudes and behaviours of different ethnic groups-to improve patient outcomes. ${ }^{26}$

\section{Strengths and limitations}

The findings are specific to the UK but appear to resonate with some US research. ${ }^{4}$ The sample was large for a qualitative study $(\mathrm{n}=94)$. However, despite this we may not have fully captured the diversity of Black African women despite reporting findings by ethnic group and generation. Variation is likely to exist between countries and regions in relation to cancer-related health education, ${ }^{11}$ awareness, beliefs, attitudes and behaviours. ${ }^{27}$ The sample size and heterogeneity of participants means the sample is unlikely to be representative and may be too small to inform. Thus, some caution should be paid to health promotion messages advocated in this paper until findings presented have been further validated.

Clinical records showed that more Black women were diagnosed with late stage disease than White women. We purposively sought to include Black as well as White women with late stage disease to understand reasons for late diagnosis. However, in the event, more Black women with advanced disease were recruited to the study, which could have impacted the findings. Further, the retrospective design of the interview phase may have hindered women's accurate recall of events.

Untangling the effects of socioeconomic factors and ethnicity is difficult. Work of others, including Schneider, clearly demonstrates the coexistence of socioeconomic factors and ethnicity and their influence over cancer staging and outcomes. ${ }^{28}$ We sought to address this issue by matching White British women with Black African and Black Caribbean women when possible. However, White British women tended to be more highly educated than other ethnic groups, which could impact on the findings.

Interview findings were strengthened by their validation using vignettes, created from the interviews, in focus groups. However, focus group data did not confirm expected differences ${ }^{4}$ between the help-seeking intention of women without breast cancer and actual help-seeking of women with breast cancer. This finding may be an artefact of sampling, may have arisen because the vignettes prompted women to think about particular barriers, and also may have resulted from inclusion of women with and without breast cancer in the same focus group or resulted from inclusion of women for whom breast cancer was particularly salient due to their friend/relative's breast cancer experiences. Family and friends without breast cancer might have felt unwilling to contradict the experiences of women with breast cancer or found their own views similar to theirs. Women who had never had a friend or family member with breast cancer may respond to breast changes differently. These women were not represented in this research.

We were aware that researchers' attitudes in qualitative research can influence design, data collection and analysis. We therefore used the steering committee to ensure a balanced representation of the data. ${ }^{29}$ In addition, the steering group contributed to understanding study findings and their implications for policy and practice.

\section{CONCLUSIONS}

Our findings suggest there are important differences in barriers to early diagnosis of breast cancer between Black African, Black Caribbean and White British women living in the UK. First generation Black African women experienced the most barriers and were therefore particularly vulnerable to delay. In contrast, second generation Black Caribbean and White British women were similar and experienced fewer barriers compared to other groups. Older White British women ( $\geq 70$ years) and first generation Black African and Black Caribbean women shared conservative attitudes and taboos about breast awareness. Women from all ethnic groups were confused about what they needed to do to be breast aware.

The treatment of Black women homogenously in the reporting of studies is very likely to mask important distinctions within and between ethnic groups. Current 
media and health promotion messages need reframing to promote early presentation. Working with communities and developing culturally appropriate materials can help break down taboos and stigma, raise awareness, increase discussion of breast cancer and promote prompt help-seeking for breast symptoms among women with lower cancer awareness. These interventions need to help women determine what changes may mean, and empower them to decide to seek help and attend healthcare appointments for early diagnosis and intervention.

\section{Author affiliations}

${ }^{1}$ King's College London, Florence Nightingale School of Nursing and Midwifery, London, UK

${ }^{2}$ National Nursing Research Unit, King's College London, Florence Nightingale School of Nursing and Midwifery, London, UK

${ }^{3}$ The Royal Marsden NHS Foundation Trust, London, UK

${ }^{4}$ Faculty of Life Sciences and Medicine, King's College London, London, UK ${ }^{5}$ National Cancer Intelligence Network, Public Health England, London, UK

${ }^{6}$ University of Surrey, School of Health Sciences, Guildford, Surrey, UK

Twitter Follow Grace Lucas at @gracerbowman

Acknowledgements The authors thank M MacKenzie (Independent Cancer Patients Voice), M Ornstein, K Scanlon (Breast Cancer Care), H Shekede, A Shewbridge (Guy's and St Thomas' NHS Foundation Trust) and R Thompson (BME Cancer Communities) for their contribution to the project as steering committee members.

Contributors ER, CELJ, JM, GL, EAD and RHJ were responsible for the study design. CELJ was responsible for data collection. CELJ, JM, GL and ER were responsible for data analysis and interpretation. All authors contributed in drafting the manuscript. ER is guarantor.

Funding This work was supported by the National Awareness and Early Diagnosis Initiative (NAEDI; grant number C7000/A12219). The NAEDI funding consortium, under the auspices of the National Cancer Research Institute (NCRI), consists of Cancer Research UK; Department of Health (England); Economic and Social Research Council; Health \& Social Care R\&D Division, Public Health Agency (Northern Ireland); National Institute for Social Care and Health Research (Wales) and the Scottish Government.

\section{Competing interests None.}

Ethics approval Ethics approval was obtained from the East of England Research Ethics Committee (MREC number 11/EE/0118) and governance approval was obtained from participating NHS Trusts. All women gave written informed consent at the beginning of the study to CELJ or clinical staff. This was confirmed verbally prior to interviews and focus groups.

Provenance and peer review Not commissioned; externally peer reviewed.

Data sharing statement No additional data are available.

Open Access This is an Open Access article distributed in accordance with the Creative Commons Attribution Non Commercial (CC BY-NC 4.0) license, which permits others to distribute, remix, adapt, build upon this work noncommercially, and license their derivative works on different terms, provided the original work is properly cited and the use is non-commercial. See: http:// creativecommons.org/licenses/by-nc/4.0/

\section{REFERENCES}

1. Ferlay J, Soerjomataram I, Ervik M, et al. GLOBOCAN 2012 v1.0, Cancer Incidence and Mortality Worldwide: IARC CancerBase No. 11 [Internet]. Lyon, France: International Agency for Research on Cancer, 2013. http://globocan.iarc.fr (accessed Jun 2014).

2. Jack RH, Davies EA, Møller H. Breast cancer incidence, stage, treatment and survival in ethnic groups in South East England. $\mathrm{Br} \mathrm{J}$ Cancer 2009;100:545-50.
3. Jack RH, Davies EA, Renshaw C, et al. Differences in breast cancer hormone receptor status in ethnic groups: a London population. Eur J Cancer 2013;49:696-702.

4. Jones $\mathrm{CEL}$, Maben J, Jack $\mathrm{RH}$, et al. A systematic review of barriers to early presentation and diagnosis with breast cancer among black women. BMJ Open 2014;4:e004076.

5. NHS Breast Screening Programme. Breast awareness. 2014. http:// www.cancerscreening.nhs.uk/breastscreen/breastawareness.html (accessed Aug 2014).

6. Weller D, Vedsted P, Rubin G, et al. The Aarhus statement: improving design and reporting of studies on early cancer diagnosis. Br J Cancer 2012;106:1262-7.

7. Charmaz K. Constructing grounded theory. London: Sage, 2006:42-96

8. Spencer L, Ritchie J, O'Connor W, et al. Analysis in practice. In: Ritchie J, Lewis J, Nicholls M, et al. eds. Qualitative research practice: a guide for social science students and researchers. London: Sage, 2013:295-343.

9. Smith LK, Pope C, Botha JL. Patients' help-seeking experiences and delay in cancer presentation: a qualitative synthesis. Lancet 2005;366:825-31.

10. Odusanya OO. Breast cancer: knowledge, attitudes and practice of female schoolteachers in Lagos, Nigeria. Breast J 2001;7:171-5.

11. Livingston J. Improvising medicine: an African oncology ward in an emerging cancer epidemic. Durham, NC: Duke University Press, 2012:1-52.

12. Dein S. Explanatory models of and attitudes towards cancer in different cultures. Lancet Oncol 2004;5:119-24.

13. Kösters JP, Gøtzsche PC. Regular self-examination or clinical examination for early detection of breast cancer. Cochrane Libr 2003;(2):CD003373.

14. NHS. Breast cancer awareness: be breast aware. http://www.nhs.uk/ Livewell/Breastcancer/Pages/Breastcancersymptoms.aspx (accessed Jan 2015)

15. Austoker J. Breast self-examination. BMJ 2003;326:1-2.

16. Chapman S, McLeod K, Wakefield M, et al. Impact of news of celebrity illness on breast cancer screening: Kylie Minogue's breast cancer diagnosis. Med J Aust 2005;183:247-50.

17. Cancer Research UK, England and Wales Survival (2010-2011) Summary-April 2014. http://publications.cancerresearchuk.org/ cancerstats/statssurvival/surv-data-table.html (accessed Aug 2014).

18. Cismaru M, Lavack AM, Hadjistavropoulos $\mathrm{H}$, et al. Understanding health behaviour: an integrated model for social marketers. Soc Mark Q 2008;14:2-32.

19. Kinney AY, Boonyasiriwat W, Walters ST, et al. Tele health personalized cancer risk communication to motivate colonoscopy in relatives of patients with colorectal cancer: the family CARE randomized controlled trial. J Clin Oncol 2014;32:654-62.

20. Kreuter MW, Lukwago SN, Bucholtz RD, et al. Achieving cultural appropriateness in health promotion programs: targeted and tailored approaches. Health Educ Behav 2003;30:133-46.

21. Lasserson DS, Chandratheva A, Giles MF, et al. Influence of genera practice opening hours on delay in seeking medical attention after transient ischaemic attack (TIA) and minor strike: prospective population based study. BMJ 2008;337:a1569.

22. Kingsland JP, Swinyard PW. Should general practices open for longer? BMJ 2013;347:f6832.

23. Ream E, Finnegan-John J, Pedersen VH. Evaluation of a community-based prostate health clinic in London, 2012. https://www. $\mathrm{kcl.ac} . u k /$ nursing/research/programmes/PatientCarerExperience/ Evalutation-of-a-Prostate-Health-Clinic---report.pdf (accessed Jun 2014).

24. Mckinstry B, Heaney D, Sheikh A. Telephone consulting in primary care: a triangulated qualitative study of patients and providers. $\mathrm{Br} J$ Gen Pract 2009;59:e209-218.

25. NHS England. Transforming primary care in England, 2013. http:// www.england.nhs.uk/london/wp-content/uploads/sites/8/2013/11/ Call-Action-ACCESSIBLE.pdf (accessed Aug 2014)

26. Betancourt JR, Green AR, Carrillo JE, et al. Defining cultural competence: a practical framework for addressing racial/ethnic disparities in health and healthcare. Public Health Rep 2003;118:293-302.

27. Azubuike S, Okwuokei S. Knowledge, attitudes and practices of women towards breast cancer in Benin City, Nigeria. Ann Med Health Sci Res 2013;3:155-60.

28. Schneider EC. Disparities and colorectal cancer. In: Koh H, ed. Toward the elimination of cancer disparities: a clinical and public health perspective. New York: Springer, 2009:161-78.

29. Malterud K. Qualitative research: standards, challenges, and guidelines. Lancet 2001;358:483-8. 